TAO, Vol. 15, No. 3, 493-502, September 2004

\title{
Statistical Assessment for the Hazard of Large Aftershocks Post to the Chi-Chi Mainshock
}

\author{
Yuh-Ing Chen ${ }^{1, *}$, Chi-Shen Huang ${ }^{1}$ and Chien-Hwei Hong ${ }^{1}$ \\ (Manuscript received 18 June 2004, in final form 28 August 2004)
}

\begin{abstract}
Real-time hazard assessment for large aftershocks from a major earthquake is usually needed for disaster relief and rescue efforts. In this paper, we employ the Reasenberg-Jones (RJ) model to describe the time-magnitude distribution of aftershocks after the Chi-Chi earthquake ( $M=7.3,1999$ / 9/21). Both the maximum likelihood estimated RJ model and the Bayesian estimated RJ model are used to compute the probability of having at least one future $M \geq 5.0$ aftershock. A probabilistic aftershock hazard map (PAHM) is also constructed for estimating the likely spatial distribution of $M \geq 5.0$ aftershocks over the study region. This map gives then alarming for potential hazardous area of future $M \geq \mathbf{5 . 0}$ aftershocks. Finally, we evaluate the association between the future $M \geq \mathbf{5 . 0}$ aftershocks and the alarming obtained from the maximum likelihood estimated PAHM and Bayesian estimated PAHM, denoted by LPAHM and BPAHM, respectively. The results suggest that the LPAHM-based alarming is superior to the BPAHM-based one for locating future $M \geq 5.0$ aftershocks after the Chi-Chi earthquake.
\end{abstract}

(Key words: Aftershock hazard, Bayesian estimate, Conjugate prior distribution, Maximum likelihood estimate)

\section{INTRODUCTION}

Real-time information about the hazard of large aftershocks is often urgent for ongoing emergency services to reduce loss of life, especially, in a short time after a disastrous mainshock over a region near the mainshock epicenter. To fulfill the purpose, Reasenberg and Jones

\footnotetext{
${ }^{1}$ Institute of Statistics, National Central University, Chung-Li, Taiwan, ROC

* Corresponding author address.Dr. Yuh-Ing Chen, Institute of Statistics, National Central University, No. 300 Junda Road, Chung-Li, Taiwan, ROC; E-mail: ychen@stat.ncu.edu.tw
} 
$(1989,1994)$ combined the modified Omori's law (Utsu 1961) and the Gutenberg-Richter (1944) relationship to describe the time-magnitude distribution of aftershocks in a specific region. The analysis of aftershocks through the Reasenberg-Jones model, referred as R-J model hereafter, is then suggested based on the maximum likelihood estimates (MLE) of the associated parameters computed from the current aftershock sequence.

When previous aftershock sequences are available, however, Reasenberg and Jones (1989) proposed to build up prior distributions of the parameters in their model. To adjust the prior information about the aftershock hazard based on current aftershocks, Reasenberg and Jones (1989) further recommend, for the California aftershock hazard, use of Bayesian estimates of the parameters in the R-J model with conjugate normal prior distributions.

Notice that the aftershock occurrence is usually heterogeneous over the study region. To portray spatial hazard of large aftershocks in a region during a time period after the mainshock, Wiemer (2000) applied the gridding technique (Wiemer and Wyss 2000) and obtained a probabilistic aftershock hazard map (PAHM) based on the maximum likelihood estimated R-J model. Wiemer (2000) also explored the rough relationship between the probability and the associated peak ground acceleration.

In this paper, we employ the dense spatial grids at nodes spaced at distance of $10 \mathrm{~km}$ in a $100 \mathrm{~km} \times 100 \mathrm{~km}$ study region near the Chi-Chi epicenter. We find the maximum likelihood estimated R-J model based on the reported $M \geq 4.0$ aftershocks in each grid centered at a node with $20 \mathrm{~km}$ in radius. We also find the Bayesian estimated R-J model in each grid based on the current Chi-Chi aftershocks and previous aftershock sequences with mainshock epicenters in the northeastern and western Taiwan. We then obtain the maximum likelihood-based and Bayesian-based probabilistic aftershock hazard maps over the study region, denoted by LPAHM and BPAHM, respectively. Moreover, based on both maps, we consider issuing alarms for $M \geq 5.0$ aftershocks in the Chi-Chi sequence. Finally, we calculate the odds ratio of the aftershocks in the alarming area for evaluating the association between the alarming area and the future $M \geq 5.0$ Chi-Chi aftershocks.

\section{MODELS AND METHODS}

In general, the frequency-magnitude relationship (Gutenberg and Richter 1944) states that the magnitude of earthquakes is distributed according to a left-truncated exponential distribution with survival function

$$
S(M)=\operatorname{Prob}\{\text { Magnitude }>M\}=\exp \left\{-\beta\left(M-M_{c}\right)\right\},
$$

where $M_{c}$ is the cut-off magnitude and $\beta$ is a constant. Notice that $\beta=\mathrm{b} \ln 10$, where $\mathrm{b}$ is the usual b-value which, in general, depends on the heterogeneous of materials (Mogi 1962) and In denotes natural logarithm. However, the occurrence of aftershocks is usually described as a non-stationary Poisson process with hazard decaying in time according to the modified Omori's law (Utsu 1961): 


$$
\lambda(t)=K /(t+c)^{p},
$$

where $\lambda(t)$ is the intensity or hazard of aftershocks at time $t$ after the mainshock, and $K, c$, and $p$ are constants. Notice that the parameter $K$ generally depends on the amount of aftershocks, while the parameter $p$ may be related to the frictional heat (Wiemer and Katsumata 1999). Furthermore, Reasenberg and Jones $(1989,1994)$, combining the modified Omori's law and the Gutenberg-Richter relationship, proposed a hazard model to describe the intensity of aftershocks with magnitude $M$ or larger at time $t$ after the mainshock as

$$
\lambda(t, M)=\lambda(t) S(M)
$$

Hence, the probability $P$ of at least one aftershock with magnitude $M$ or larger occurring in the time period $\left(T_{1}, T_{2}\right)$ after the mainshock is given by

$$
P=1-\exp \left[S(M) \int_{T_{1}}^{T_{2}} \lambda(t) d t\right]
$$

Suppose that the records of a sequence of aftershocks with different occurrence times and magnitudes are $\left\{\left(t_{i}, M_{i}\right): M_{i} \geq M_{c}, 0<t_{i}<\mathrm{T}, \mathrm{i}=1,2, \ldots, \mathrm{n}\right\}$. Then, the MLE of $(K, c, p)$ can be obtained by using an iteration algorithm in Ogata $(1983,1988)$ and the MLE of $\beta$ is given by $\left(\bar{M}-M_{c}\right)^{-1}$, where $\bar{M}=\sum_{i=1}^{n} M_{i} / n$ (Gutenberg and Richter 1944). Therefore, the probability $P$ in (4) can be estimated, denoted by $P_{L}$, by replacing the parameters $K, c, p$ and $\beta$ with the related MLE.

However, to make a near real-time assessment for aftershock distribution based on both the previous aftershock sequences and the available current aftershock data, Reasenberg and Jones (1989) suggested a Bayesian estimated RJ model. Notice that the maximum likelihood estimated R-J model was obtained for each of the 62 previous aftershock sequences in California with mainshock magnitude 5.5 or larger. The empirical distribution of each involved MLE suggests the normal prior distribution with mean $\theta_{0}$ and variance $\sigma_{0}^{2}$ for each parameter $\theta$, that is $K, c, p$ or $\beta$, in the R-J model. Reasenberg and Jones (1989) then obtained Bayesian estimated R-J model based on the conjugate normal prior distributions. In other words, the Bayesian estimate of each parameter is the mean of the associated posterior distribution given by

$$
\theta_{b}=\frac{s^{2}}{s^{2}+\sigma_{0}^{2}} \theta_{0}+\frac{\sigma_{0}^{2}}{s^{2}+\sigma_{0}^{2}} \hat{\theta}
$$

and the posterior variance is 


$$
s^{2} \sigma_{0}^{2} /\left(s^{2}+\sigma_{0}^{2}\right),
$$

where $\hat{\theta}$ is the MLE of $\theta$ and $s^{2}$ is the estimated variance of $\hat{\theta}$. Replacing the Bayesian estimates of $K, c, p$ and $\beta$ into the probability $P$ in (4), we then obtain the Bayesian estimated probability of at least one aftershock with magnitude $M$ or larger occurring in a time period, denoted by $P_{B}$.

\section{DATA ANALYSIS AND RESULTS}

We investigate the hazard of $\mathrm{M} \geq 5.0$ aftershocks post to the Chi-Chi earthquake ( $M=7.3$, $\left.1999 / 9 / 21,23.85^{\circ} \mathrm{N}, 120.78^{\circ} \mathrm{E}\right)$ in the region $\left(23.4^{\circ} \mathrm{N}, 24.4^{\circ} \mathrm{N}\right) \times\left(120.5^{\circ} \mathrm{E}, 121.5^{\circ} \mathrm{E}\right)($ Fig. 1$)$. We compute the minimum magnitude for complete reporting, denoted by $M_{c}$, of Chi-Chi aftershocks from the Central Weather Bureau (CWB) in the study region based on the $90 \%$ goodness-of-fit for the Gutenberg-Richter relationship (Wiemer and Wyss 2000). The results show that the minimum magnitude is less than 3.05 and apparently decreases in time (Fig. 2a).
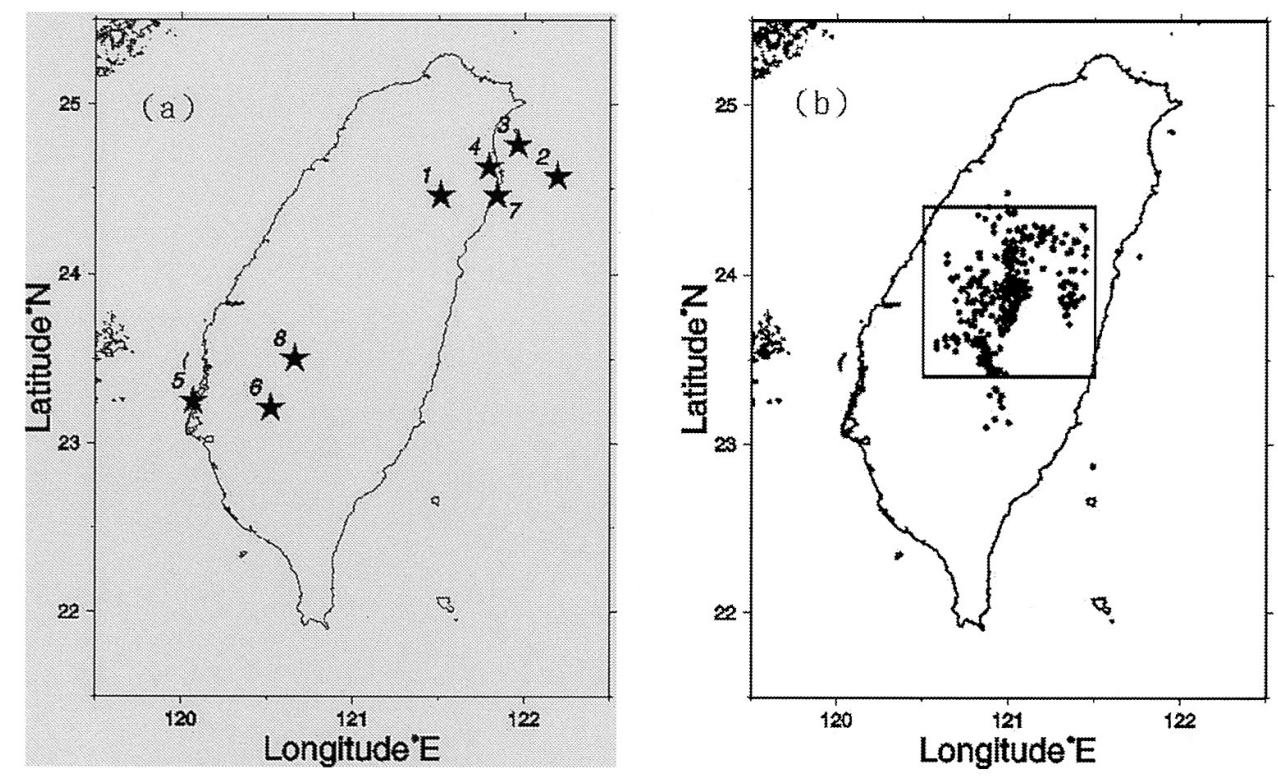

Fig. 1. Previous 8 aftershock sequences and the Chi-Chi aftershocks. (a) Black stars 1-8 locate the epicenters of previous mainshocks during 1983 - 1998. (b) Black dots represent $M \geq 4.0$ aftershocks within 7 days after the ChiChi earthquake in the study region covering the rectangular area of $\left(23.4^{\circ} \mathrm{N}, 24.4^{\circ} \mathrm{N}\right) \times\left(120.5^{\circ} \mathrm{E}, 121.5^{\circ} \mathrm{E}\right)$. 
Moreover, applying a two-dimensional gridding over the study region where overlapping 20$\mathrm{km}$-radius circles have centers located on a grid with $10-\mathrm{km}$ in distance, we obtain, in total, 121 circles. A survey of spatial-varying minimum magnitude of complete record of aftershocks in $\mathrm{T}$ days after the Chi-Chi earthquake over the gridding region (Fig. 2b) further indicates that, for $\mathrm{T}=2,5$ and 7 , the available minimum magnitudes are all less than 4.0. Notice that, in practice, only large aftershocks are available in a short time after a major earthquake. Therefore, we used herein the $M \geq 4.0$ aftershocks to fit the R-J model for a more reliable near real-time assessment of $M \geq 5.0$ Chi-Chi aftershock hazard. Notice that, when the number of recorded aftershocks is less than 30 or the $90 \%$ goodness-of-fit is not reachable, we simply ignore the corresponding grids for future study.

To obtain a priori information about the distribution of Chi-Chi aftershocks, a survey of the earthquake catalog during 1983 - 1998 from the CWB in Taiwan yields 8 distinct aftershock sequences marked by their mainshock epicenters in Fig. 1. We also identified the $M_{c}$ for each of the 8 aftershock sequence based on, again, the $90 \%$ goodness-of-fit for the GutenbergRichter relationship (Wiemer and Wyss 2000). The R-J model was then fitted to each aftershock sequence and the mean and variance of the 8 MLE for each parameter were reported in Table 1 . These mean and variance are finally taken to be the parameters, $\theta_{0}$ and $\sigma_{0}^{2}$, in the normal prior distribution.

The maximum likelihood estimated R-J models were computed based on the current $M \geq 4.0$ aftershocks available at day $\mathrm{T}$ after the Chi-Chi mainshock, for $\mathrm{T}=2,3,5$ and 7 . The
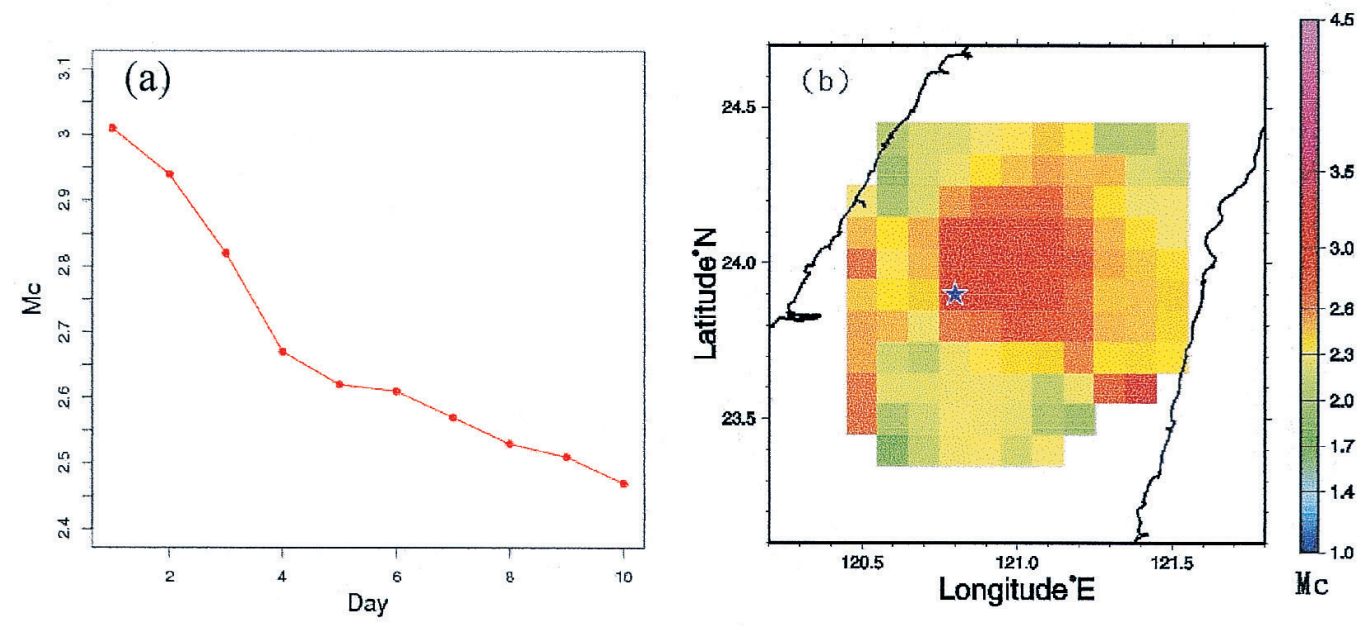

Fig. 2. The minimum magnitude of the complete reported Chi-Chi aftershocks. (a) The time varying of the minimum magnitude of the complete report Chi-Chi aftershocks in the study region. (b) The spatial varying of the minimum magnitude of the complete report aftershocks in 5 days after the Chi-Chi earthquake, where the blue star locates the epicenter of the Chi-Chi earthquake. 
Bayesian estimated RJ models were then obtained by the input of relevant MLE and the prior normal parameters into (5) and (6). All the estimated parameters and the associated standard deviation, as well as the probability, $P_{L}$ and $P_{B}$, of the occurrence of at least one $\mathrm{M} \geq 5.0$ aftershock in the following week are finally reported in Table 2.

Table 1. The maximum likelihood estimates of the parameters in the ReasenbergJones models for previous complete aftershock sequences during 19831998.

\begin{tabular}{cccccccc}
\hline No. & Time & $\mathrm{Mm}$ & $\mathrm{Mc}$ & $\mathrm{k}$ & $\mathrm{c}$ & $\mathrm{p}$ & $\beta$ \\
\hline 1 & $1983 / 05 / 10$ & 6.00 & 3.31 & 1.86 & 0.01 & 1.11 & 2.04 \\
2 & $1985 / 06 / 12$ & 6.09 & 3.65 & 4.826 & 0.01 & 0.79 & 2.20 \\
3 & $1986 / 01 / 16$ & 6.09 & 1.80 & 98.05 & 0.03 & 0.77 & 1.67 \\
4 & $1986 / 07 / 30$ & 5.82 & 1.85 & 52.45 & 0.06 & 1.17 & 2.28 \\
5 & $1991 / 03 / 12$ & 5.67 & 2.93 & 26.14 & 0.20 & 0.94 & 1.33 \\
6 & $1993 / 12 / 15$ & 5.70 & 2.87 & 2.77 & 0.00 & 1.02 & 1.71 \\
7 & $1994 / 06 / 05$ & 6.50 & 3.95 & 4.75 & 0.02 & 0.98 & 2.25 \\
8 & $1998 / 07 / 17$ & 6.20 & 2.15 & 16.39 & 0.01 & 1.09 & 1.49 \\
\hline Mean & & & & 25.90 & 0.04 & 0.98 & 1.87 \\
Variance & & & & 1142.4 & 0.005 & 0.023 & 0.137 \\
\hline
\end{tabular}

Table 2. The estimated parameters and the associated standard errors in the Reasenberg-Jones model for $M \geq 4.0$ aftershocks of size $\mathrm{n}$ in T days after the Chi-Chi earthquake.

\begin{tabular}{lccccc}
\hline T days / $\mathrm{n}$ & Method & $\mathrm{k}$ & $\mathrm{c}$ & $\mathrm{p}$ & $\beta$ \\
\hline \multirow{2}{*}{2 days / 321 } & MLE & $98.9 \pm 12.3$ & $0.14 \pm 0.10$ & $1.3 \pm 0.2$ & $1.9 \pm 0.1$ \\
& Bayesian & $90.4 \pm 11.5$ & $0.09 \pm 0.04$ & $1.1 \pm 0.1$ & $1.9 \pm 0.1$ \\
\multirow{3}{*}{ days / 341 } & MLE & $100.7 \pm 16.2$ & $0.19 \pm 0.10$ & $1.4 \pm 0.2$ & $2.0 \pm 0.1$ \\
& Bayesian & $86.7 \pm 14.6$ & $0.10 \pm 0.05$ & $1.1 \pm 0.1$ & $2.0 \pm 0.1$ \\
5 days / 374 & MLE & $97.0 \pm 12.4$ & $0.15 \pm 0.10$ & $1.3 \pm 0.1$ & $2.0 \pm 0.1$ \\
& Bayesian & $88.6 \pm 11.7$ & $0.11 \pm 0.04$ & $1.2 \pm 0.1$ & $2.0 \pm 0.1$ \\
7 days / 401 & MLE & $94.8 \pm 10.4$ & $0.13 \pm 0.04$ & $1.2 \pm 0.1$ & $2.0 \pm 0.1$ \\
& Bayesian & $88.9 \pm 9.9$ & $0.10 \pm 0.04$ & $1.2 \pm 0.1$ & $2.0 \pm 0.1$ \\
\hline
\end{tabular}


To further investigate the spatial distribution of $M \geq 5.0$ Chi-Chi aftershocks over the study region, we estimated the R-J model based on the $M \geq 4.0$ aftershocks in each related grid within the first $\mathrm{T}$ days after the Chi-Chi earthquake. The corresponding probabilities, $P_{L}$ and $P_{B}$, were then computed for evaluating the hazard of $M \geq 5.0$ aftershocks occurring in the period of $(\mathrm{T}, \mathrm{T}+7)$ days, for T $=2,3,5$ and 7 . The LPAHM and BPAHM based on $P_{L}$ and $P_{B}$, respectively, were finally constructed for mapping the likely spatial distribution of $M \geq 5.0$ aftershocks in the following one week for $\mathrm{T}=2,3,5$ and 7 . Figure 3 shows, in particular, the LPAHM and BPAHM for $\mathrm{T}=5$. Notice that we computed the probability only for the grids with at least 20 aftershocks of magnitude 4.0 or larger.
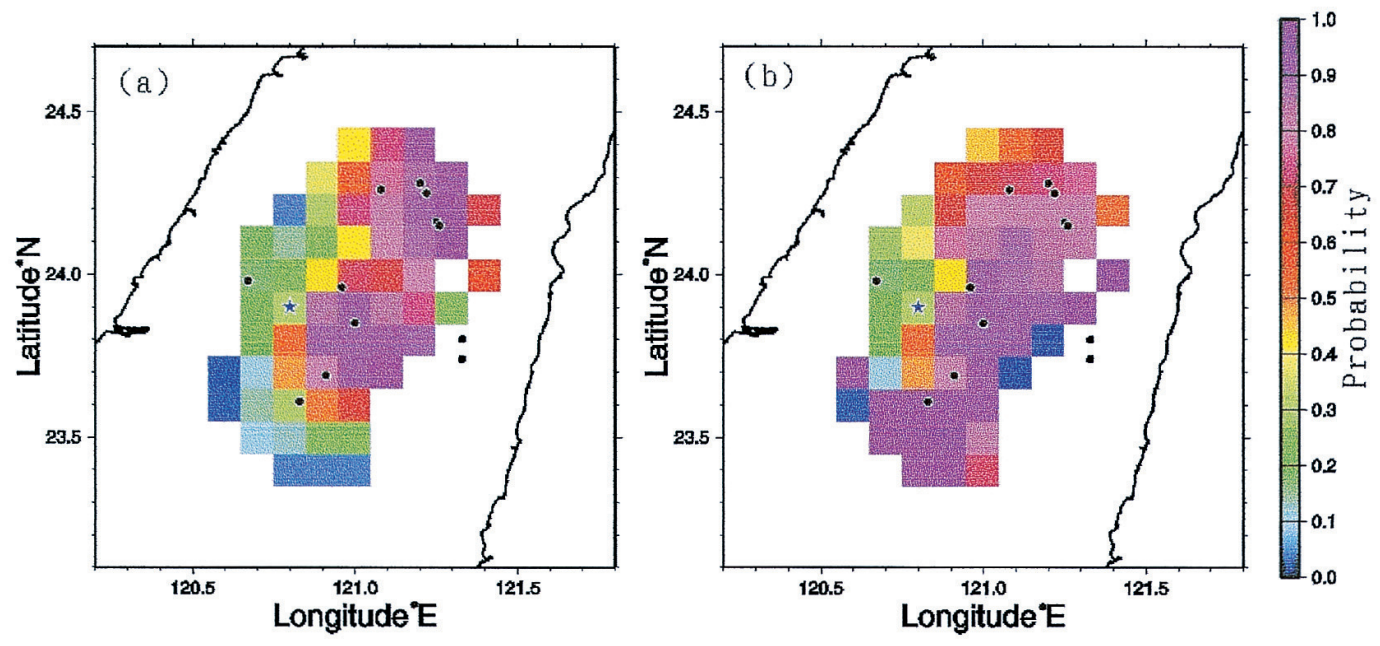

Fig. 3. The (a) LPAHM and (b) BPAHM constructed at day 5 after the Chi-Chi earthquake for $M \geq 5.0$ aftershocks in the following week. The blue star locates the epicenter of the Chi-Chi earthquake and the black dots denote the $M \geq 5.0$ aftershocks during 5 - 12 days after the Chi-Chi earthquake.

We consider set up the alarm of the hazardous area for future large aftershocks by choosing a reasonable probability threshold, denoted by $P_{0}$, on the available probabilistic aftershock hazard map. A future $M \geq 5.0$ aftershock is then classified into the alarming area, if its epicenter is relatively closed to the node where the associated grid has the probability higher than the given probability threshold. If, however, the closest node corresponds to a grid with probability lower than the given probability threshold, the aftershock is then assigned into the nonalarming area. Finally, we computed the odds of future $M \geq 5.0$ aftershocks in the alarming and non-alarming areas, respectively. The ratio of the two odds, termed conventionally as odds ratio of $M \geq 5.0$ aftershocks in the alarming area for any given $P_{0}$ between $0.50-0.80$ 
were then obtained (Fig. 4). For example, using the LPAHM-based alarming with $P_{0}=0.70$, the odds ratio of 9.5 indicates that the future $M \geq 5.0$ aftershocks has 9.5 times more frequently to occur in the alarming area than does in the non-alarming area during $5-12$ days to the ChiChi earthquake.

Notice that the LPAHM-based odds ratios are larger than 5 at $P_{0}$ between $0.70-0.80$ for $\mathrm{T}=2$ and 5, and are saturated between 5.0 and 6.0 at $P_{0} \geq 0.65$ for $\mathrm{T}=3$, but when $\mathrm{T}=7$, the associated odds ratio drops down to 2.2 at $P_{0}=0.70$. The BPAHM-based odds ratio for $\mathrm{T}=2$ increases in $P_{0}$ and attains it maximum of 5.1 at $P_{0}=0.80$. When $\mathrm{T}=3$, the BPAHM-based odds ratios lie between 5.0 and 6.0 for $0.70 \leq P_{0} \leq 0.75$. When $\mathrm{T}=5$, the maximum BPAHMbased odds ratio of 7.1 occurs at $P_{0}=0.70$, but the odds ratio decreases to 2.3 at $P_{0}=0.75$. However, for $\mathrm{T}=7$, the maximum of the BPAHM-based odds ratio appears at $P_{0}=0.60$, the odds ratio then drops down to about 3.9 at $P_{0}=0.65$, and finally turns up to about 8.0 at $P_{0}=0.80$. As a comparison between the LPAHM and BPAHM, Figure 5 indicates that the LPAHM-based odds ratio is larger than the corresponding BPAHM-based one for $\mathrm{T}=2$ and 5 .
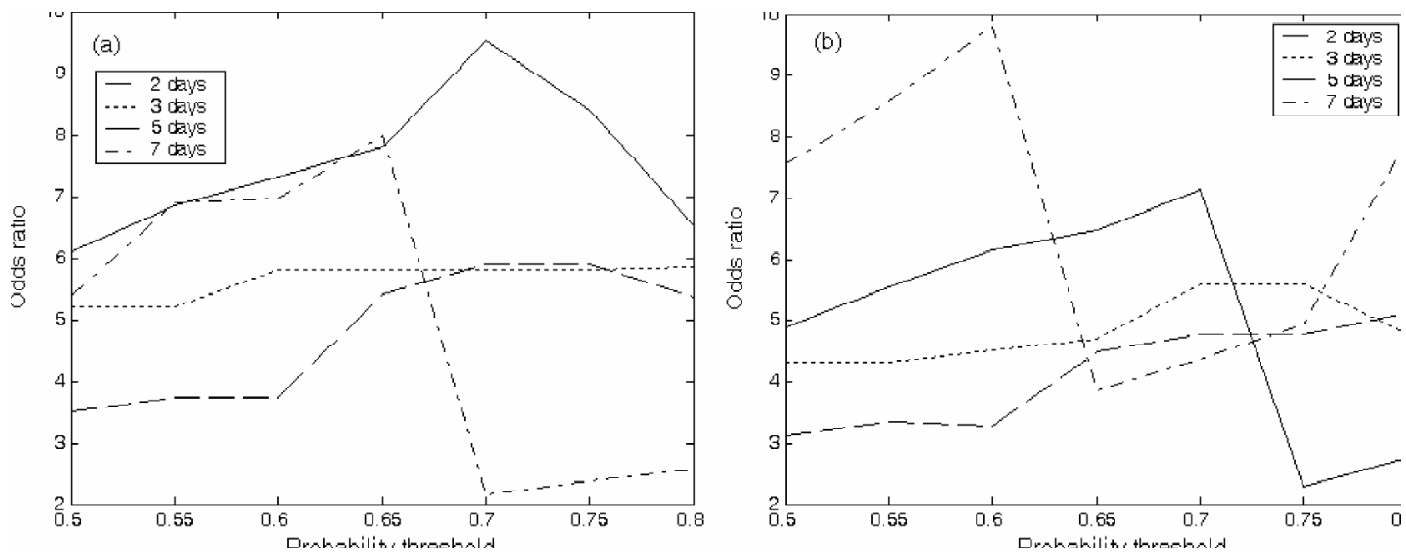

Fig. 4. The odds ratios of $M \geq 5.0$ Chi-Chi aftershocks in the probability threshold-based alarming areas based on (a) LPAHM and (b) BPAHM, respectively.

\section{DISCUSSIONS AND CONCLUSIONS}

The probabilistic aftershock hazard maps based on both the Bayesian estimated and the maximum likelihood estimated R-J models are constructed in a short time after the Chi-Chi earthquake. These maps, reflecting somehow the local structures, are of practical use since they describe the likely spatial distribution of the large aftershocks in a very short time post to an important mainshock. Therefore, we suggest alarming future large aftershocks on the basis of these probabilistic aftershock hazard maps with reasonable probability threshold. 

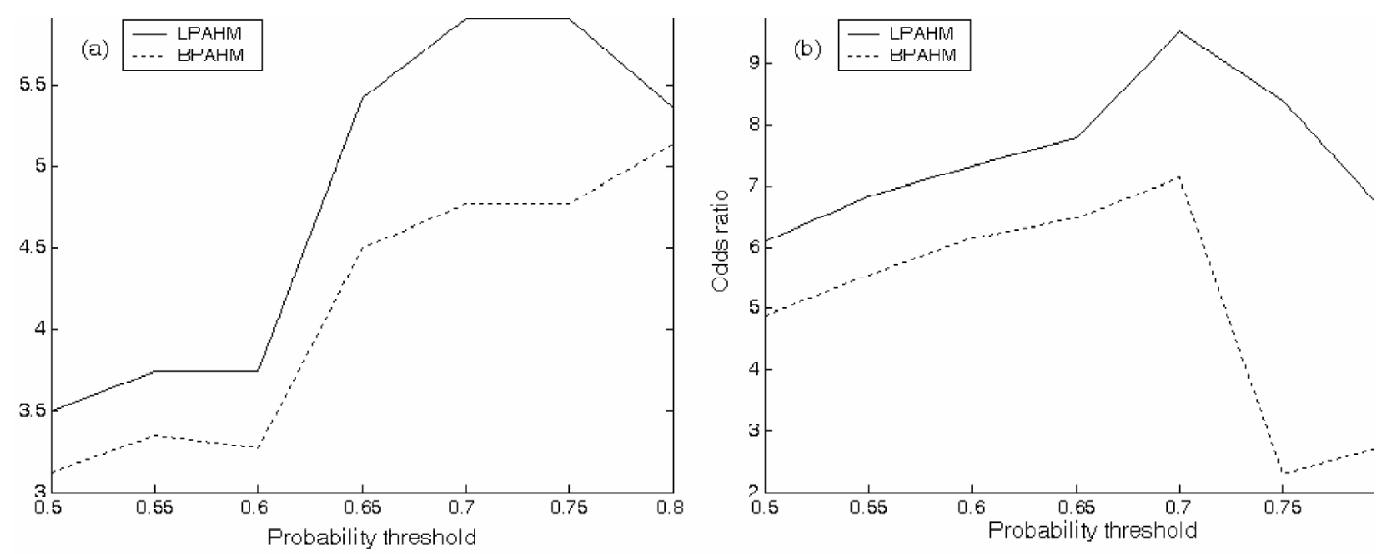

Fig. 5. The odds ratios of $M \geq 5.0$ aftershocks in the probability threshold-based alarming areas based on LPAHM and BPAHM, respectively, at (a) day 2 and (b) day 5 after the Chi-Chi earthquake.

According to the analysis of the $M \geq 4.0$ aftershocks post to the Chi-Chi earthquake, it is found that the probabilistic aftershock hazard map based on the maximum likelihood estimated R-J model, or LPAHM, is, in general, better than the BPAHM for locating $M \geq 5.0$ ChiChi aftershocks in short terms. This may be due to the unreasonable assumption about the normal time-magnitude distribution of aftershocks inherent into the Bayesian analysis based on normal conjugate prior distribution (Rydelek 1990). In fact, for the 8 aftershock sequences in the Taiwan area, the p-values of the Anderson-Darling normal tests for the parameters $K, c$, $p$ and $\beta$ are $0.018,0.001,0.515$ and 0.305 , respectively. The testing results indicate that the normal distribution assumption are reasonable for the parameters $p$ and $\beta$, but it is not suitable for the parameters $K$ and $c$. Therefore, when more current aftershocks are introduced into the Bayesian analysis, the more evident we have for the non-normal time-magnitude distribution of the aftershocks and, hence, the worse the BPAHM for alarming the following $M \geq 5.0$ ChiChi aftershocks.

Finally, the odds ratio of the $M \geq 5.0$ aftershocks in the alarming area suggests, within one week after the Chi-Chi earthquake, use of probability threshold of $0.65-0.80$ is reasonable for alarming the $M \geq 5.0$ aftershocks in the following week based on the LPAHM. However, alarming the $M \geq 5.0$ aftershocks in the following week at day 7 after the Chi-Chi earthquake based on the LPAHM, it would be better to use the probability threshold between 0.55-0.65.

Acknowledgements This research was supported by the Ministry of Education, ROC under the grant 甲-91-N-FA07-7-4, and by the National Science Council under the grant NSC892921-M-008-018-EAF. The authors would like to thank the referees for their comments and suggestions that improve the presentation of this paper. 


\section{REFERENCE}

Gutenberg, R., and C. F. Richter, 1944: Frequency of earthquakes in California. Bull. Seism. Soc. Am., 34, 185-188.

Mogi, K., 1962: Magnitude-frequency relation for elastic shocks accompanying fractures of various materials and some related problem in earthquakes. Bull. Earthquake Res. Inst. Univ. Tokyo, 40, 831-853.

Ogata, Y., 1983: Estimation of the parameters in the modified Omori formula for aftershock sequences by the maximum likelihood procedure. J. Phys. Earth, 31, 115-124.

Ogata, Y., 1988: Statistical models for earthquake occurrences. J. Am. Stat. Assoc., 83, 9-27.

Reasenberg P. A., and L. M. Jones, 1989: Earthquake hazard after a mainshock in California. Science, 243, 1173-1176.

Reasenberg P. A., and L. M. Jones, 1994: Earthquake aftershocks: update. Science, 265, 12511252.

Rydelek, P. A., 1990: California aftershock model uncertainties. Science, 247, 343.

Utsu, T., 1961: Statistical study on the occurrence of aftershocks. Geophys. Mag., 30, 521605.

Wiemer, S., 2000: Introducing probabilistic aftershock hazard mapping. Geophys. Res. Lett., 27, 3405-3408.

Wiemer, S., and K. Katsumata, 1999: Spatial variability of seismicity parameters in aftershock zones. J. Geophys. Res., 104, 13,135-13,151.

Wiemer, S., and M. Wyss, 2000: Minimum magnitude of completeness in earthquake catalogs: Examples from Alaska, the western US and Japan. Bull. Seism. Soc. Am., 90, 859-869. 